\title{
Bartholin's Gland Cyst or Abscess: A Novel Surgical Treatment Using a Looped Packing Strip a Case Report
}

\author{
David Hobson ${ }^{1}$ \\ ${ }^{1}$ Spring Branch Community Health Center
}

June 25, 2020

Title

Bartholin's Gland Cyst or Abscess: A Novel Surgical Treatment Using a Looped Packing Strip: a case report Author

David W. Hobson, MD, American Board of Obstetrics and Gynecology, Fellow American College of Obstetricians and Gynecologists

Department of Obstetrics and Gynecology, Spring Branch Community Health Center, Houston Texas

Correspondence

Spring Branch Community Health Center 800 West Sam Houston Parkway South Suite 200 Houston Texas 77042-1914 Email: dhobson@sbchc.net

Comments

This case report is submitted to BJOG An International Journal of Obstetrics and Gynaecology in response to the paper written by Illingworth BJG, Stocking K, Showell M, Kirk E, Duffy JMN. Evaluation of treatments for Bartholin's cyst or abscess: a systematic review. BJOG 2020;127:671-678.

Illustrations(Figures)

Title

Bartholin's Gland Cyst or Abscess: A Novel Surgical Treatment using a Looped Packing Strip: a case report

Keywords: Bartholin's Cyst, Bartholin's Abscess, Word Catheter, case report, packing strip

Abstract

This is the first reported surgical treatment of a Bartholin's cyst under local anesthesia using a looped plain packing strip passed through two incisions of the Bartholin's cyst or abscess, loosely tied and left in place four to six weeks creating a permanent fistula. The potential advantages of this procedure include: 1) ease of use 2) low cost 3) lower risk for recurrences 4) requires minimal training 5) alternative choice in patients who would like to avoid the use of a catheter and other more invasive treatments 6 ) use in cysts smaller than $2 \mathrm{~cm}$ in size.

Introduction

The Bartholin's gland produces mucoid secretions that provide lubrication of the distal vagina and labia minora. The Bartholin's duct commonly becomes obstructed leading to the formation of a cyst or abscess. This affects about $2 \%$ of women mostly of reproductive age11Omole F, Simmons DJ, Hacker Y. Management of Bartholin's duct cyst and gland abscess. Am Fam Physician 2003;68:135-40. Patients often complain of 
pain in the vulva when sitting, walking and during intercourse. They are seen in many clinical settings such as emergency rooms, outpatient clinics and Doctors' offices. Treatments have included aspiration, incision and drainage, packing the abscess, insertion of catheters, rings, silver nitrate gland ablation, marsupialization, sclerotherapy, resection of the gland,and carbon dioxide laser procedures. Systematic reviews22Illingworth BJG, Stocking K, Showell M, Kirk E, Duffy JMN. Evaluation of treatments for Bartholin's cyst or abscess: a systematic review. BJOG 2020;127:671-678 have not identified the best approach. Currently, placement of the Word catheter under local anesthesia is the favored procedure.33Krose JA, van der Velde M, Morssink LP, Zafarmand MH, Geomini P, van Kesteren P, et al. Word catheter and marsupialization in women with a cyst or abscess of the Bartholin gland (WoMan-trial): a randomized clinical trial. BJOG 2017:124:243-9. However the use of the Word catheter has several limitations such as: 1) balloon loss reported as high as 43\%, ${ }^{2} 44$ Reif, P, Ulrich D, Bjelic-Radisic V, Hausler M, Schnedl-Lampprecht E, Tamussino K. Management of Bartholin's cyst and abscess using the Word catheter: implemenatation, recurrence, rates and costs. Eur J Obstet Gynecol Reprod Biol 2015;190:81-84 2) technical challenges such as catheter leak, deflation, inflating the catheter with a needle larger than 24 gauge, failure to direct the needle into the lumen of the catheter, failure to maintain pressure on the syringe plunger as you remove the needle from the inflated catheter, and vulvar pain from the inflated catheter,55Mellick L. A word about Word Catheters. 2012 Aug 1. Available from: https://journals.lww.com/em_news/pages/blogs.aspx 3) recurrences of the cyst or abscess ranging from 4-17\%66Wechter ME, Wu JM, Marzano D, Haefner H. Management of Bartholin duct cysts and abscesses. A systematic review.Obstet Gynecol Surv 2009;64:395-404. Figure 1. Bartholin's cysts with open ostia

The WoMan-trial (Word versus Marsupialization) was a randomized clinical trial that compared treatment of a Bartholin's cyst or abscess under local or no anesthesia to marsupialization performed under spinal or general anesthesia. The recurrence of a cyst or abscess, median time for recurrence, and postoperative pain scores after 24 hours were not significantly different between the two groups. Kroese et al concluded that that treatment using a Word catheter was a faster, lower cost alternative, and relieved pain sooner after diagnosis compared to marsupialization. ${ }^{3}$

Randomized clinical trials to date have not identified the best treatment of a Bartholin's cyst or abscess. The best approach will be the development of a novel intervention with devices that are smaller in size, more comfortable, remains in place four to six weeks, and creates a permanent fistula. ${ }^{2}$

Materials and Methods

A 45 year old female was seen in our clinic during the period of May 2018 through May 2020 by the author. Written informed consent was obtained for treatment and publication. There was no financial or other incentive for participation. There were no sources of support and no conflicts of interest. She complained of a six year history of swelling in the right labia minora. Physical examination confirmed a $4 \mathrm{~cm}$ right Bartholin's cyst. One week later she presented with a $6 \mathrm{~cm}$ right and $4 \mathrm{~cm}$ left Bartholin's cyst. Both cysts were incised and drained. She had recurrence of both cysts documented on visit day 15 . On visit day 15 , the right Bartholin's cyst was incised, drained with placement of the looped packing strip. The left Bartholin's cyst was $4 \mathrm{~cm}$ and not drained. The packing strip was removed 11 days later. The second recurrence of the right Bartholin's cyst was 10 days after removal of the packing strip. On visit day 36 she had a $4 \mathrm{~cm}$ right Bartholin's and $10 \mathrm{~cm}$ left Bartholin's cyst. Both Bartholin cysts were incised, drained, and treated with insertion of bilateral packing strips that remained in place for 32 days. Incision and drainage consisted of a sterile prep using povidone iodine 10\% solution, local anesthesia using Lidocaine 1\% 3ml, 25 gauge 5/8 inch needles, with injections into the labial skin overlying the cyst 1-2 cm outside of the introitus, and making one $5 \mathrm{~mm}$ stab incision with a no. 11 blade scalpel. The insertion of the packing strip consisted of making two $5 \mathrm{~mm}$ stab incisions with a no. 11 blade scalpel through the skin and cyst wall, under local anesthesia, one at the top and bottom edges of the cyst 1-2 cm lateral to the introitus. The tip of a curved hemostat was inserted into the lower incision passing through the upper incision. A $\frac{1}{2}$ inch $\times 15 \mathrm{~cm}$ length plain packing strip was pulled through both incisions and loosely tied using a square knot. Allowance was made to avoid tension on the skin. The extra packing strip was cut close to the knot. Postoperative instructions included 
twice daily cleansing with a gentle wash and returning to the clinic in 4-6 weeks for removal of the packing strip.

\section{Results}

The patient was contacted by telephone and agreed to come in for evaluation. Informed consent was obtained for publication. The patient was seen by the author in our clinic 1.72 years after placement of the looped plain strips that remained in place for 32 days. Examination confirmed that both Bartholin cysts were draining mucoid secretions through two ostia. (fig.1)

\section{Discussion}

This case report demonstrates how the treatment with incision and drainage alone and removal of the packing strip 11 days after insertion resulted in the recurrence of a right Bartholin's cyst. Treatment using packing strip left in place for 32 days resulted in the creation of permanent fistulas. Both cysts were noted to drain through created ostia seen 1.72 years after removal of the looped plain packing strip. The primary outcome measure in the treatment of a Bartholin's cyst or abscess is the creation of a permanent fistula. The preferred treatment of a Bartholin's cyst or abscess should be fast, safe, performed in an outpatient setting under local anesthesia, heal rapidly without complication, and have low recurrence. ${ }^{2}$ This procedure requires minimal training, is cost effective, and well tolerated by this patient. The novel placement of the looped packing strip in this patient with bilateral recurrent Bartholin's cysts accomplished this goal. Further research is needed to compare placement of the looped packing strip versus insertion of the Word catheter.

Disclosure of interests: there were no financial, personal, political, intellectual or religious interests

Contribution to authorship: there is only one author David W. Hobson, MD

Patient's consent: consent for evaluation, treatment, procedures and publication was obtained in writing

Funding: none required

References

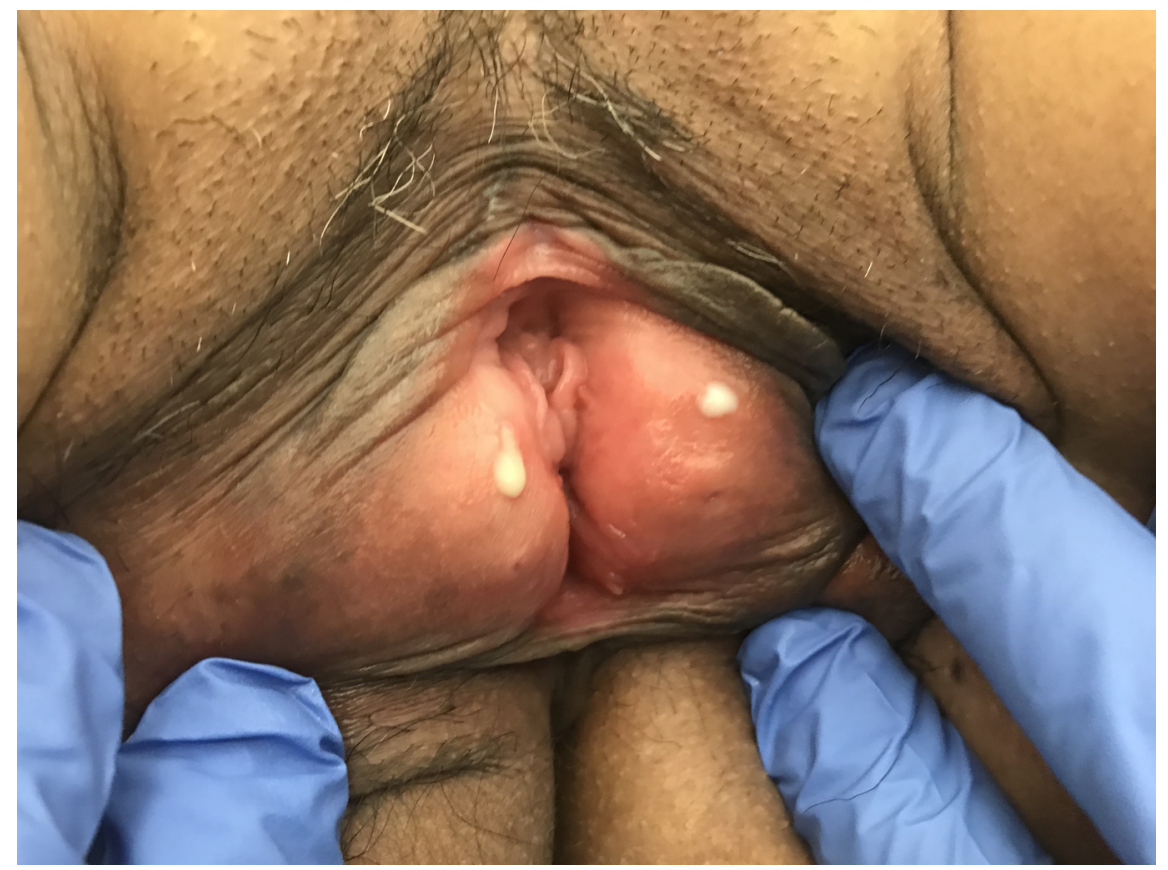

